EGU2020-22108

https://doi.org/10.5194/egusphere-egu2020-22108

EGU General Assembly 2020

(c) Author(s) 2020. This work is distributed under

the Creative Commons Attribution 4.0 License.

\title{
Evolution of the atmospheric organic content on Titan with seasons
}

\author{
Athena Coustenis ${ }^{1}$, Donald Jennings ${ }^{2}$, Richard Achterberg ${ }^{2}$, Panayotis Lavvas ${ }^{3}$, Conor Nixon ${ }^{2}$, F. \\ Michael Flasar ${ }^{2}$, and Georgios Bampasidis ${ }^{4}$ \\ 1'LESIA, Paris Observatory, CNRS, PSL, Meudon, France (athena.coustenis@obspm.fr) \\ ${ }^{2}$ Detector Systems Branch, Goddard Space Flight Center, Greenbelt, MD 20771, USA \\ ${ }^{3}$ Université de Reims Champagne-Ardennes, CNRS, GSMA, UMR 7331, 51097 Reims, France \\ ${ }^{4}$ School of Education, Department of Primary Education, National \& Kapodistrian University of Athens, 10680 Athens, \\ Greece
}

Titan is one of the most promising bodies in the solar system from the astrobiological perspective in particular because of its large organic content in the atmosphere and on the surface. These chemical species evolve with time. We performed an analysis of spectra acquired by Cassini/CIRS at high resolution which cover the far-IR range from 10 to $1500 \mathrm{~cm}-1$ since the beginning and until the last year of the Cassini mission in 2017 and describe the temperature and composition variations near Titan's poles and at the equator over almost two Titan seasons ([1-3]. By applying our radiative transfer code (ARTT) to CIRS data and to the 1980 Voyager 1 flyby values inferred from the re-analysis of the Infrared Radiometer Spectrometer (IRIS) spectra, as well as to the intervening ground- and space-based observations (such as with ISO), we study the stratospheric evolution over a Titanian year (V1 encounter $L s=9^{\circ}$ was reached in mid-2010) $[1,2]$. CIRS nadir and limb spectral together show variations in temperature and chemical composition in the stratosphere during the Cassini mission, before and after the Northern Spring Equinox (NSE) and also during one Titan year.

After the 2010 equinox we have thus reported on monitoring of Titan's stratosphere near the poles and in particular on the observed strong temperature decrease and compositional enhancement above Titan's southern polar latitudes since 2012 and until 2014 of several trace species, such as complex hydrocarbons and nitriles, which were previously observed only at high northern latitudes. This effect followed the transition of Titan's seasons from northern winter in 2002 to northern summer in 2017, while at that latter time the southern hemisphere was entering winter.

Our data show a continued decrease of the abundances which we first reported to have started in 2015. The 2017 data we have acquired and analyzed here are important because they are the only ones recorded since 2014 close to the south pole in the far-infrared nadir mode at high resolution. A large temperature increase in the southern polar stratosphere (by $10-50 \mathrm{~K}$ in the $0.5 \mathrm{mbar}-0.05$ mbar pressure range) is found and a change in the temperature profile's shape. The 2017 observations also show a related significant decrease in most of the abundances which must have started sometime between 2014 and 2017 [3]. In our work, we show that the equatorial latitudes 
remain rather constant throughout the Cassini mission.

We have thus shown that the south pole of Titan is now losing its strong enhancement, while the north pole also slowly continues its decrease in gaseous opacities. It would have been interesting to see when this might happen, but the Cassini mission ended in September 2017. Perhaps future ground-based measurements can pursue this investigation and monitor Titan's atmosphere to characterize the seasonal events. We have obtained thus significant results which set constraints on GCM and photochemical models.

[1] Coustenis et al., 2016, Icarus 270, 409-420; [2] Coustenis et al., 2018, Astroph. J., Lett., 854, no2; [3] Coustenis et al., 2019, Icarus in press, https://doi.org/10.1016/j.icarus.2019.113413. 